\title{
7-T MRI tracking of mesenchymal stromal cells after lung injection in a rat model
}

\author{
Stefania Rizzo ${ }^{1,2,3^{*}}$ (D) Francesco Padelli ${ }^{4}$, Elena Rinaldi ${ }^{5}$, Daniela Gioeni ${ }^{6}$, Domenico Aquino ${ }^{4}$, Stefano Brizzola ${ }^{7}$, \\ Fabio Acocella ${ }^{7}$, Lorenzo Spaggiari ${ }^{8,9}$, Fulvio Baggi ${ }^{5}$, Massimo Bellomi9 ${ }^{9}{ }^{10}$, Maria Grazia Bruzzone ${ }^{11}$ and \\ Francesco Petrella $8,9,12$
}

\begin{abstract}
Background: Mesenchymal stromal cells (MSCs) are able to migrate and engraft at sites of inflammation, injuries, and tumours, but little is known about their fate after local injection. The purpose of this study is to perform MSC tracking, combining in vivo 7-T magnetic resonance imaging (MRI) and histological assessment, following lung injection in a rat model.

Methods: Five lungs were injected with ferumoxide-labelled MSCs and five with perfluorocarbon-labelled MSCs and underwent 7-T MRI. MRI acquisitions were recorded immediately $\left(T_{0}\right)$, at $24 \mathrm{~h}\left(\mathrm{~T}_{24}\right)$ and/or $48 \mathrm{~h}\left(\mathrm{~T}_{48}\right)$ after injection. For each rat, labelled cells were assessed in the main organs by MRI. Target organs were harvested under sterile conditions from rats sacrificed 0, 24, or $48 \mathrm{~h}$ after injection and fixed for histological analysis via confocal and structured illumination microscopy.

Results: Ferumoxide-labelled MSCs were not detectable in the lungs, whereas they were not visible in the distant sites. Perfluorocarbon-labelled MSCs were seen in 5/5 injected lungs at $T_{0,}$ in $1 / 2$ at $T_{24}$, and in $1 / 3$ at $T_{48}$. The fluorine signal in the liver was seen in $3 / 5$ at $T_{0}$, in $1 / 2$ at T24, and in $2 / 3$ at $T_{48}$. Post-mortem histology confirmed the presence of MSCs in the injected lung.

Conclusions: Ferumoxide-labelled cells were not seen at distant sites; a linear decay of injected perfluorocarbon-labelled MSCs was observed at $T_{0}, T_{24}$, and $T_{48}$ in the lung. In more than half of the experiments, perfluorocarbon-labelled MSCS scattering to the liver was observed, with a similar decay over time as observed in the lung.
\end{abstract}

Keywords: Ferumoxides, Fluorocarbons, Lung, Magnetic resonance imaging, Mesenchymal stromal cells

\section{Keypoints}

- Perfluorocarbon-labelled mesenchymal stromal cells (MSCs) can be detected by magnetic resonance imaging (MRI) when injected in the lungs.

- Ferumoxide-labelled MSCs cannot be detected by MRI when injected in the lungs.

\footnotetext{
* Correspondence: stefania.rizzo@eoc.ch

${ }^{1}$ Imaging Institute of the Southern Switzerland (IIMSI), Ente Ospedaliero

Cantonale (EOC), via Tesserete 46, 6900 Lugano, Switzerland

${ }^{2}$ Facoltà di Scienze biomediche, Università della Svizzera italiana (USI), Via G.

Buffi 13, 6904 Lugano, Switzerland

${ }^{3}$ Clinica di Radiologia EOC, Istituto di Imaging della Svizzera Italiana (IIMSI),

via Tesserete 46, 6900 Lugano, Switzerland

Full list of author information is available at the end of the article
}

- Perfluorocarbon-labelled MSCs injected in the lungs demonstrated some scattering to the liver.

\section{Background}

The term "regeneration" is used to describe the process in humans, whereby lost specialised tissue is replaced by proliferation of undamaged specialised cells [1]. In this regard, the main goal of regenerative medicine is to regenerate damaged tissues or whole organs by the provision of cells, as stem cells, that can stimulate wider regeneration [2]. Among the various stem cell populations used for cell therapy, adult mesenchymal stromal cells (MSCs) have emerged as a major new technology with many potential clinical applications [3]. MSCs are a population of undifferentiated multipotent adult cells that naturally reside

\section{Springer Open}

() The Author(s). 2020 Open Access This article is licensed under a Creative Commons Attribution 4.0 International License, which permits use, sharing, adaptation, distribution and reproduction in any medium or format, as long as you give appropriate credit to the original author(s) and the source, provide a link to the Creative Commons licence, and indicate if changes were made. The images or other third party material in this article are included in the article's Creative Commons licence, unless indicated otherwise in a credit line to the material. If material is not included in the article's Creative Commons licence and your intended use is not permitted by statutory regulation or exceeds the permitted use, you will need to obtain permission directly from the copyright holder. To view a copy of this licence, visit http://creativecommons.org/licenses/by/4.0/. 
within the human body and are generally defined as plastic adherent, fibroblast-like cells possessing extensive selfrenewal properties and the potential to differentiate in vivo and in vitro into a variety of mesenchymal lineage cells [4]. It has been previously demonstrated that MSCs can play an effective reparative role both in the experimental and clinical scenario of post-resection airway tissue defects $[5,6]$. Furthermore, they can act as carriers for antineoplastic drug loading and delivery, as shown in experimental cellular models [7-11]; they are able to migrate and engraft at sites of inflammation, injuries, and tumours, and they can show local reparative properties via the paracrine secretion of soluble factors $[2,12]$.

Magnetic resonance imaging (MRI) is considered an excellent method for tracking MSCs in vivo and in vitro [13]. To date, while there are limitations for the clinical use of iron-oxide agents for clinical MRI, many iron-containing compounds have been used for preclinical studies. For example, mouse bone marrow-derived endothelial progenitor cells have been labelled with ferucarbotran, and the in vitro protocol for labelling did not impair proliferative ability [14]. Choi et al. labelled human MSCs with ultrasmall paramagnetic iron oxides (USPIO) and green fluorescence protein (GFP) and demonstrated that labelled MSCs transplanted into the portal veins of immunosuppressed, hepatic-damaged rat models caused signal loss in the liver of transplanted cells in the early period of transplantation $[15,16]$. Limitations with tracking iron-labelled cells arise from low specificity, due to other regions in the image with low signal, as the lung parenchyma, and from difficulties to in vivo quantification of the signal loss.

As an alternative to iron cell tracking, fluorine-19 $\left({ }^{19} \mathrm{~F}\right)$ MRI with perfluorocarbon nanoemulsions has been used for cell tracking $[17,18]$. Its advantage is that the level of background ${ }^{19} \mathrm{~F}$ signal in host tissue is virtually absent [15]. The ${ }^{19} \mathrm{~F}$ nucleus is particularly suitable for labelling as its relative MRI sensitivity is only $17 \%$ less than that of ${ }^{1} \mathrm{H}$. Previous studies have demonstrated effective labelling of MSCs with Celsense ATM DM Red [19]. Different tracking agents and techniques have been studied, disclosing different properties and performances depending on the anatomic districts to be investigated [20-25]. With regard to airways and lungs, it has been previously demonstrated on a cellular model, that the use of two different contrast media, USPIO and perfluorocarbon (PFC) are effective for MSC labelling and MRI tracking [12].

The purpose of this study was to perform MSC tracking, combining in vivo MRI and histological assessment, following injection of MSCs into the lungs in a rat model.

\section{Methods}

\section{Animal model}

Ten Fischer 344 rats between 10 and 15 weeks old, weighing between 300 and $350 \mathrm{~g}$ were studied, bought by the Charles River Laboratories Italia s.r.l, Calco (IT), were included in this study. The animal procedures were performed in accordance with the Italian laws on animal protection (Authorisation released by the Ministry of Health, n.21/2017-PR released on January 16th 2017, according to the article n.31 of the Law n.26/2014). Animals were maintained on a 12/12-h light/dark cycle at $21 \pm 2^{\circ} \mathrm{C}$ with $50-60 \%$ humidity. Food and water were available ad libitum.

\section{MSC culture}

The MSCs were purchased from Cyagen $\left(\right.$ OriCell $^{\mathrm{TM}}$ Fischer 344 Rat Mesenchymal Stromal Cells (MSCs)/ GFP, RAFMX-01101) and grown at $37^{\circ} \mathrm{C}$ and $5 \% \mathrm{CO}_{2}$ using the MSC growth medium kit (Cyagen, GUXMX90011), consisting of the basal medium, foetal bovine serum (10\%), penicillin-streptomycin solution (1\%), and glutamine (1\%). The culture medium was changed every 3 days, and cells were passed when $80-90 \%$ confluence was reached. MSCs were derived from bone marrow tissue of F344 rats, and they transiently express the GFP.

\section{MSC labelling with Molday ION Rhodamine B and Cell Sense}

Molday ION Rhodamine B (MIRB) (BioPal Inc, Worcester, MA, USA) is an USPIO MRI contrast agent conjugated with rhodamine $\mathrm{B}$ that allows visualisation by fluorescent microscopy imaging. Cell Sense Red (CS ATM DM Red, Celsense, Inc., Pittsburgh, PA, USA) is a perfluorocarbon-based emulsion conjugated with a red fluorescent dye.

MSCs at $80 \%$ confluence were harvested with trypsinethylenediaminetetraacetic acid (ECB3052D, Euroclone S.p.A., Pero, Milan, Italy) centrifuged $(250 \times g$ for $5 \mathrm{~min})$, and the number of MSCs and their viability (Number of viable cells / [Number of dead cells + Number of viable cells], expressed as \%) were evaluated with the Trypan blue exclusion method, and cell count was performed on a haemocytometer. MSCs were seeded $\left(12 \times 10^{6}\right.$ cells $)$ in a T75 flask and, after the overnight culture, were incubated with MIRB $(50 \mu \mathrm{g} / \mathrm{mL})$ for $24 \mathrm{~h}$ or with Cell Sense Red $(20 \mathrm{mg} / \mathrm{mL})$ for $4 \mathrm{~h}$ at $37^{\circ} \mathrm{C}$ and $5 \% \mathrm{CO}_{2}$. The excess of tracers was removed with phosphate saline buffer (PBS, 3 washes). MIRB-labelled MSCs (MIRB-MSCs) and Cell Sense-labelled MSCs (CS-MSCs) were harvested with trypsin-ethylenediaminetetraacetic acid and centrifuged, and cell viability was assessed via the Trypan blue exclusion method. Stained MSCs were resuspended in PBS, diluted $1: 5$ by mixing with $0.4 \%$ Trypan blue solution, and unstained (viable) and stained (non-viable) cells were counted by means of a haemocytometer. Labelling efficiency was assessed on 4\% paraformaldehyde-fixed MIRB-MSCs and CS-MSCs by confocal microscopy, and images were analysed with Fiji 
software. Nuclei were stained with 4',6-diamidino-2phenylindole (DAPI) (Thermo Fisher Scientific). Aliquots of MIRB-MSCs and CS-MSCs $\left(2 \times 10^{6}\right.$ in $0.1 \mathrm{~mL}$ of PBS) were prepared for the transthoracic intercostal injection in the rat lung and to assess the viability and labelling stability in vitro for $48 \mathrm{~h}$.

Labelled MIRB-MSCs and CS-MSCs were cultured for $48 \mathrm{~h}$ in duplicate to assess their in vitro viability.

\section{Anaesthesia and MSC injection}

Preventive analgesia was obtained by a subcutaneous injection of Carprofen $(5 \mathrm{mg} / \mathrm{kg})$. Transthoracic intercostal injection of $2 \times 10^{6}$ MSCs suspended in $0.1 \mathrm{~mL}$ of PBS was performed in the right lung. The rat then underwent MRI under general anaesthesia, maintained until the end of the exam.

Anaesthesia was different between groups based on the type of contrast media. This differentiation was necessary for the group that received perfluorocarbonlabelled cells. Indeed, isoflurane contains fluorine, and this can create confounding findings on ${ }^{19} \mathrm{~F}$ MRI, especially within the lungs.

Accordingly, five rats were injected with MIRB-MSCs and were initially anaesthetised with isoflurane (IsoFlo $100 \%$, Zoetis Italia S.r.l., Rome, Italy) (4\% induction and $1.8-2.2 \%$ maintenance) delivered in a mixture of air and oxygen (30/70) via a nose cone. Exhaled gas from the rats was actively vacuumed away from the nose cone via a built-in vacuum line.

Five rats were injected with CS-MSCs and were induced and maintained with a mixture of dexmedetomidine (Dexdomitor $0.5 \mathrm{mg} / \mathrm{mL}$, Vetoquinol Italia, Bertinoro (FC), Italy) $200 \mathrm{mcg} / \mathrm{kg}$ and tiletamine/zolazepam (Zoletil $50 / 50 \mathrm{mg} / \mathrm{mL}$, Virbac Italia, Milan, Italy) 4 $\mathrm{mg} / \mathrm{kg}$ IP. A mixture of air and oxygen (30/70) was delivered until the end of the exam via a nose cone. The injection was well tolerated by all the animals both during the procedure, under general anaesthesia, and during/after recovery.

All procedures were performed under spontaneous ventilation. Heart rate, respiratory frequency as well as rectal temperature were monitored continuously during the procedures (Small Animals Instruments Incorporated, NY, USA). The animals' temperature was kept at $36.5 \pm 0.5^{\circ} \mathrm{C}$ by means of a warm-water circuit integrated into the animal holder.

\section{MRI protocol and image analysis}

MRI experiments were performed by a horizontal-bore 7-T preclinical scanner (BioSpec 70/20 USR, Bruker, Ettlingen, Germany), with a $20-\mathrm{cm}$ bore diameter, equipped with an actively shielded gradient system with integrated shims set up to 2nd order. The maximum gradient amplitude was $440 \mathrm{mT} / \mathrm{m}$. All acquisitions were carried out using a transceiver double-tuneable ${ }^{1} \mathrm{H} /{ }^{19} \mathrm{~F}$ linear birdcage radiofrequency coil with an inner diameter of $72 \mathrm{~mm}$. Since the whole thoraco-abdominal region shifts according to the respiratory cycle, to avoid image blurring and misalignment, MRI acquisitions were gated recording signals only during the maximum expiration phase (Small Animal Monitoring and Gating System, Small Animals Instruments Incorporated, NY, USA).

\section{${ }^{7} \mathrm{H} M R I$}

MIRB belongs to the USPIO contrast agent family. It has a colloidal size of $35 \mathrm{~nm}$, a zeta potential of about +31 $\mathrm{mV}$, and an iron concentration of $2 \mathrm{mg} / \mathrm{mL}$. Beside the cross-linked red fluorescence dye, MIRB is a contrast agent that shortens the T2 value of neighbouring protons. MIRB therefore appears dark in images where nanoparticles accumulate. T2-weighted rapid acquisition with refocused echo (RARE) sequences were acquired on the abdominal region (Fig. 1) of MIRB-MSC-injected animals. MRI sequence parameters are reported in Table 1. The animals' anaesthesia protocol and physiological parameters monitoring were carried out as reported above.

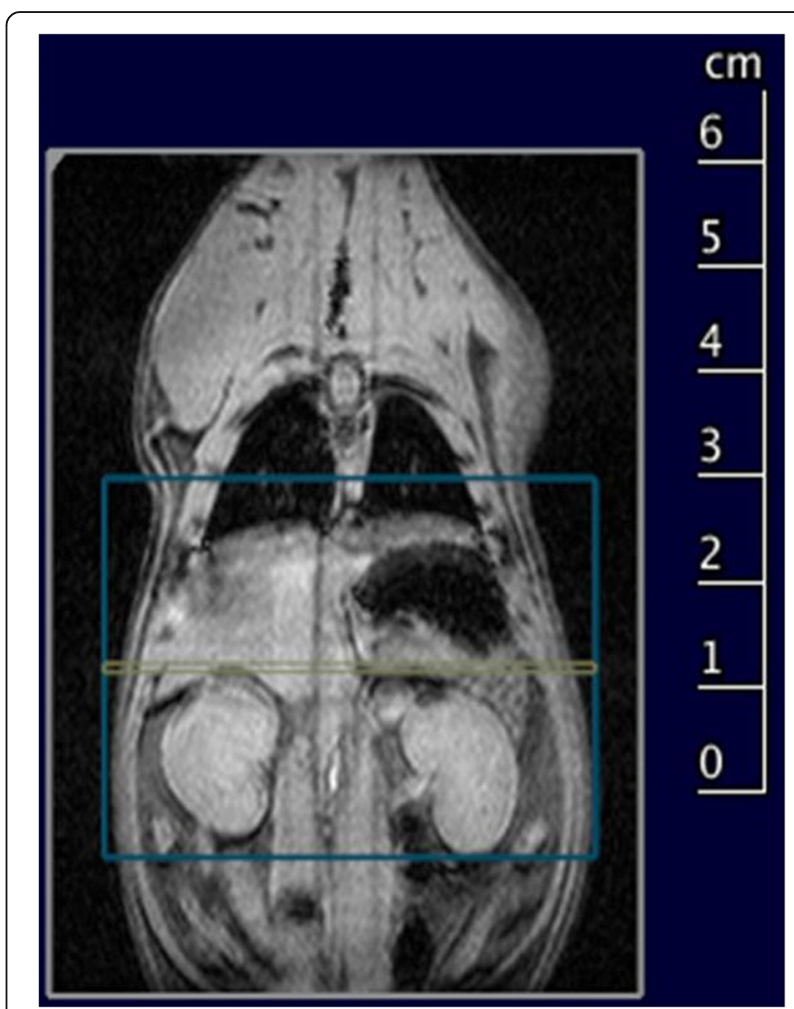

Fig. 1 Slice geometry of the ${ }^{1} \mathrm{H}$ T2-weighted rapid acquisition with refocused echo (RARE) sequence visualised onto a multiplane multislice localiser sequence of a non-injected animal 
Table 1 Acquisition parameters of ${ }^{1} \mathrm{H}$ and ${ }^{19} \mathrm{~F}$ sequences

\begin{tabular}{lll}
\hline & 1 H MRl & ${ }^{\mathbf{1 9}} \mathrm{F} \mathrm{MRl}$ \\
\hline Echo time (ms) & 27 & 6.5 \\
Repetition time (ms) & 4,050 & 2,000 \\
RARE factor & 8 & 8 \\
Flip angle (degrees) & $90-180$ & $90-180$ \\
Read field of view & 50 & 60 \\
Phase field of view & 35 & 52.5 \\
Slice geometry & Contiguous axial & Contiguous axial \\
Number of slices & 46 & 20 \\
Slice thickness (mm) & 0.8 & 3 \\
Read matrix size & 200 & 40 \\
Phase matrix size & 140 & 35 \\
k-space encoding order & Linear & Centric \\
Resonance frequency (MHz) & 300.324 & 282.559 \\
Number of excitations & 30 & 250 \\
Acquisition time (min) & 34 & 33 \\
\hline
\end{tabular}

MRI Magnetic resonance imaging, RARE Rapid acquisition with refocused echoes

\section{${ }^{19} \mathrm{~F} \mathrm{MRI}$}

Cell Sense is a linear PFC-based emulsion with total fluorine content of $120 \mathrm{mg} / \mathrm{mL}$. Being conjugated with a green fluorescent dye, Cell Sense is available as ${ }^{19} \mathrm{~F}$ MRI contrast agent and detectable using conventional fluorescence detection modalities. The protocol for ${ }^{19} \mathrm{~F}$ MRI was optimised for the best sensitivity from the fluorine signal, setting the correct excitation frequency, optimising the excitation bandwidth and the pulse gain. ${ }^{19} \mathrm{~F}$ RARE sequences were acquired on the thoracoabdominal region (Fig. 2) of CS-MSC-injected animals at 0,24 , and $48 \mathrm{~h}$ after inoculation. MRI sequence parameters are reported in Table 1. For anatomical localisation purposes, after ${ }^{19} \mathrm{~F}$ MRI, a conventional ${ }^{1} \mathrm{H}$ T2-weighted RARE sequence was acquired. Both fluorine and proton sequences were acquired in the same geometry without changing the radiofrequency coil or moving the animal. No co-registration processes were therefore necessary to correctly locate the signals. For each ${ }^{19} \mathrm{~F}$ MRI-imaged animal, signal-to-noise ratio (SNR) of visible organs was estimated with respect to muscular tissue, which seems not to take up the labelled cells. A region of interest (ROI) was manually drawn on the detectable signal, while another ROI was delineated in the back muscle region of the animal. SNR was then evaluated as the ratio between signal ROI mean pixel value and muscle ROI standard deviation.

For each MRI examination, a radiologist with 15 years of experience in MRI reading, evaluated all the images in order to assess the presence of labelled MSCs. For MIRB-MSCs, the T2-weighted RARE images were evaluated (Fig. 3); for CS-MSCs, the fluorine sequences were

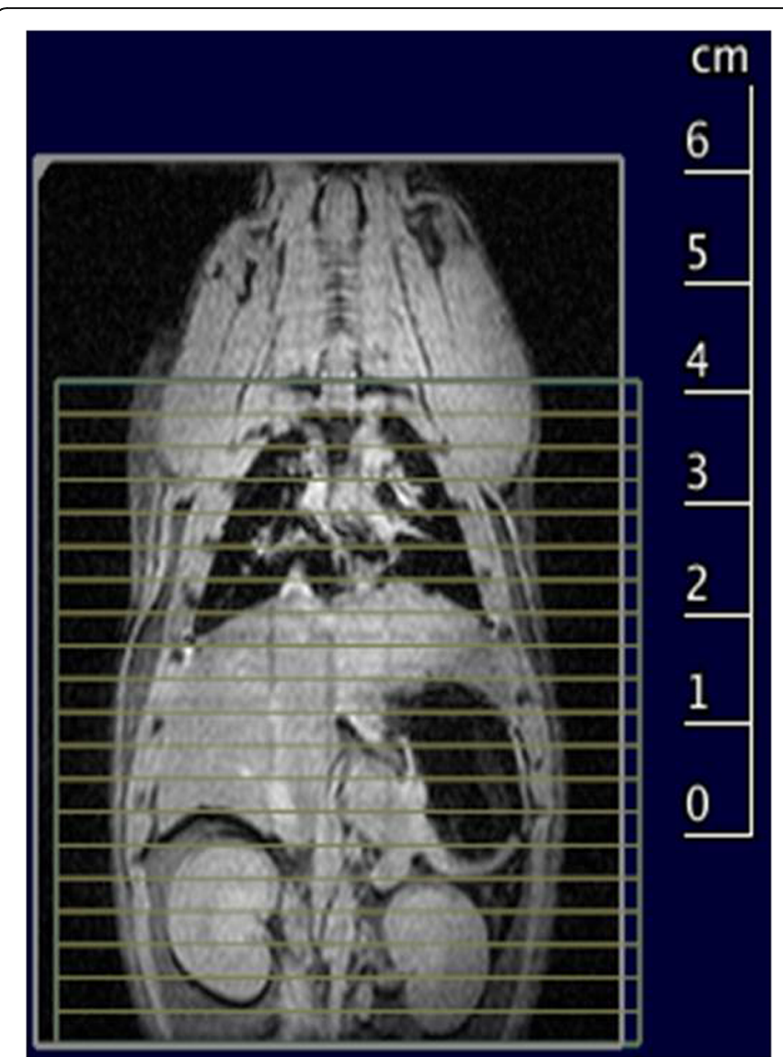

Fig. 2 Slice geometry of the ${ }^{19} \mathrm{~F}$ rapid acquisition with refocused echo (RARE) sequence visualised onto a multiplane multislice localiser sequence of a non-injected animal

evaluated along with the proton T2-weighted RARE sequences (Fig. 4), for localisation purposes. For each rat, the time of MRI acquisition was recorded according to the following times: $\mathrm{T}_{0}=$ acquisition right after the injection, $\mathrm{T}_{24}=$ acquisition $24 \mathrm{~h}$ after injection, $\mathrm{T}_{48}=$ acquisition $48 \mathrm{~h}$ after injection. For each MRI, the following sites (if included in the acquisitions, as described above) were assessed for the presence of labelled cells: right lung, left lung, heart, liver, spleen, pancreas, adrenal glands, kidneys, and other sites, if any.

In order to follow the $3 \mathrm{R}$ principle (replacement, reduction, refinement), we used the animals as controls for themselves. Therefore, all the animals $(n=10)$ underwent MRI after the injection of the labelled cells. In the original project, we had planned to sacrifice 1 rat at each time point, but in the MIRB-labelled group, one rat died after the sacrifice of the first one. Therefore, the numbers of the rats imaged at MRI and sacrificed at the following time points were different for the 2 groups, as synthesised in Table 2.

\section{Histopathology}

After MRI analyses, the animals were sacrificed and lungs removed for histological studies. The apical part of 


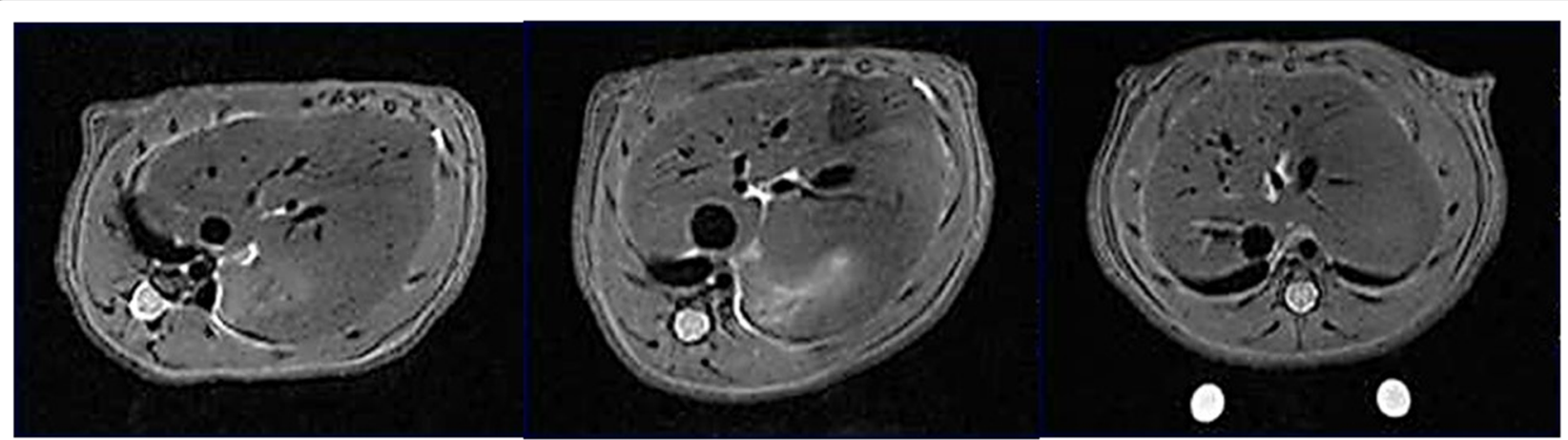

Fig. $3{ }^{1} \mathrm{H}$ magnetic resonance imaging of ferumoxides mesenchymal stromal cell-injected animals at $\mathrm{T}_{0}$ (left), $\mathrm{T}_{24}$ (centre), and $\mathrm{T}_{48}$ (right) after inoculation. Liver slices are shown. The intrahyperintense signal at $\mathrm{T}_{24}$ is due to water content in the animal stomach

the right lung was entirely sectioned in a coronal manner, and $\sim 160$ sections/rat were analysed via confocal microscopy. Lung sections were evaluated for the presence of GFP-MSCs labelled with Cell Sense.

Lungs were removed under sterile conditions from rats sacrificed at 0,24 , or $48 \mathrm{~h}$ to evaluate the presence of labelled MSCs. Lung lobes were then separated, washed with PBS containing 1\% penicillin-streptomycin, fixed in $4 \%$ paraformaldehyde in PBS and then in 30\% sucrose for $24 \mathrm{~h}$ for cryopreservation, embedded in Killik (BioOptica, Milan, Italy) and stored at $-80^{\circ} \mathrm{C}$. The cranial and middle lobes of the injected right lung were fully processed, along with the apical part of the contralateral lung. Firstly, serial tissue slices $(10-\mu \mathrm{m}$ thick) were sectioned, and every fifth section was counterstained with DAPI and examined for detection of green signals $(\mathrm{GFP}+)$ and red signals (USPIO+ and $\mathrm{PFC}+$ ) within lung, by means of a fluorescence microscopy (Nikon Eclipse TE2000-E, objective 20×, Nikon, Tokyo, Japan) equipped with B-2A filter for GFP signal and tetramethylrhodamine filter for USPIO and PFC signals. Then, single-plan and $z$-scan images of lung sections with MSCs were acquired via confocal microscopy (Nikon Eclipse TE2000-E, 20 $\times$ and $40 \times$ objectives, Nikon, Tokyo, Japan) and structured illumination microscopy (100× APOTIRF objective, Nikon, Tokyo, Japan). Liver and spleen were processed as previously stated and sectioned in 15$\mu \mathrm{m}$ thick serial tissue slices. At least 120 sections for the liver and 30 sections for the spleen were examined for detection of fluorescence signal, via fluorescence microscopy. To further investigate the Cell Sense labelling, a higher resolution analysis was conducted on selected slices via structured illumination microscopy.

\section{Results}

MRI

Among the five rats that received injection of MIRBMSCs, five were imaged by MRI at $\mathrm{T}_{0}$; three were imaged at $\mathrm{T}_{24}$; two were imaged at $\mathrm{T}_{48}$. One rat died after the first MRI and after the planned sacrifice of the first rat; therefore, the number of rats imaged at $48 \mathrm{~h}$ was two, instead of three. As mentioned, the lungs were not

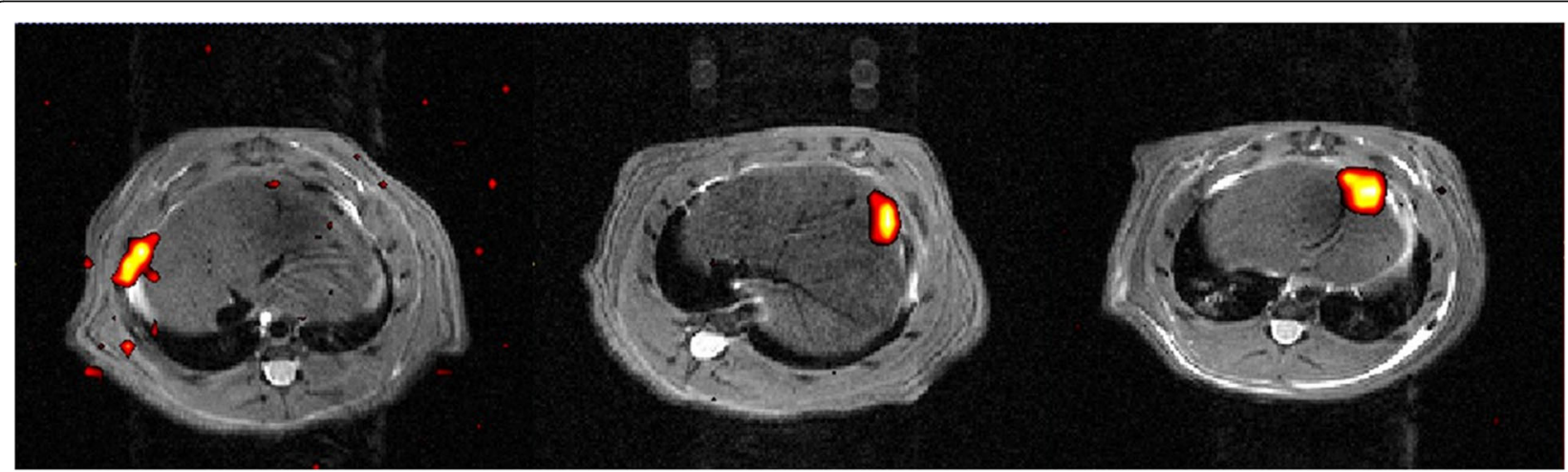

Fig. $4{ }^{19} \mathrm{~F}$ magnetic resonance imaging (MRI) of Cell Sense ATM DM Red mesenchymal stromal cell-injected animals at To (left), T24 (centre), and $\mathrm{T}_{48}$ (right) after inoculation. Red-scale images are ${ }^{19} \mathrm{~F}$ MRI while underlying greyscale images are ${ }^{1} \mathrm{H}$ anatomical MRI. Slices corresponding to the maximum ${ }^{19} \mathrm{~F}$ signal localisation are shown. 
Table 2 Number of rats imaged at magnetic resonance imaging and sacrificed (in parentheses) at different time points after injection of labelled mesenchymal stromal cells

\begin{tabular}{llll}
\hline & \multicolumn{3}{l}{ Number of rats } \\
\cline { 2 - 4 } & $\mathrm{T}_{0}$ & $\mathrm{~T}_{24}$ & $\mathrm{~T}_{48}$ \\
\hline MIRB-labelled MSCs & $5(2)$ & $3(1)$ & $2(2)$ \\
CS-labelled MSCs & $5(1)$ & $2(1)$ & $3(3)$ \\
\hline
\end{tabular}

MIRB Molday lon Rodhamine B, MSCs Mesenchymal stromal cells, CS Cell Sense

assessed in this group because of the intrinsic limitation of the T2-weighted sequences in evaluating aircontaining structures. Evaluation of distant organs did not show any signal clearly due to MIRB-MSCs at any time point.

Among the five rats that received injection of CSMSCs, five were imaged by MRI at $\mathrm{T}_{0}$, two were imaged at $\mathrm{T}_{24}$, and three were imaged at $\mathrm{T}_{48}$. The fluorine signal was seen in $5 / 5$ injected lungs at $T_{0}$, in $1 / 2$ injected lungs at $T_{24}$, and in $1 / 3$ injected lungs at $T_{48}$. Furthermore, the fluorine signal was seen in $3 / 5$ livers of the rats at $T_{0}$, in $1 / 2$ livers of the rats at $T_{24}$, and in $2 / 3$ livers of the rats at $\mathrm{T}_{48}$ (Table 3 ). No fluorine signal was seen in the other organs assessed. In one rat, there was some fluorine signal at $\mathrm{T}_{48}$ along the pleura ipsilateral to the injection, whereas in the same rat there was no remnant of fluorine signal in the lung at $\mathrm{T}_{48}$.

Table 4 shows average SNR values among all examined animals' lung and liver signal. Ratios of the evaluations at $\mathrm{T}_{24}$ and $\mathrm{T}_{48}$ with respect to the initial time point $\mathrm{T}_{0}$ are also reported.

\section{MSC detection and histopathology}

In this study, we observed that the USPIO labelling efficacy in vivo was similar to a previous published paper, regarding labelling efficacy in vitro [12]. For the PFC labelling, we tested two concentrations $(10 \mathrm{mg} / \mathrm{mL}$ and $20 \mathrm{mg} / \mathrm{mL}$ ), and we detected a fluorine signal in MSCs incubated with the latter dose.

After MIRB labelling, 100 cells were viable out of 114 total cells (87.7\%); MIRB labelling was repeated twice, and viability was $83.3 \%$ and $85 \%$ (range $83.3-87.7 \%$ ). Cell viability after CS labelling showed 92 viable cells out of

Table 3 Magnetic resonance imaging findings in the lung and liver of CS-labelled mesenchymal stromal cells

\begin{tabular}{llll}
\hline & $\mathrm{T}_{\mathbf{0}}$ & $\mathrm{T}_{\mathbf{2 4}}$ & $\mathrm{T}_{\mathbf{4 8}}$ \\
\hline Lung & & & \\
$\quad$ Number & $5 / 5$ & $1 / 2$ & $1 / 3$ \\
$\quad$ Percentage & $100 \%$ & $50 \%$ & $33 \%$ \\
Liver & & & \\
$\quad$ Number & $3 / 5$ & $1 / 2$ & $2 / 3$ \\
$\quad$ Percentage & $60 \%$ & $50 \%$ & $66 \%$ \\
\hline CS Cell Sense & & &
\end{tabular}

CS Cell Sense
Table 4 Signal-to-noise ratios (SNR) averaged among all examined animals after the injection of perfluorocarbon-labelled mesenchymal stromal cells

\begin{tabular}{llllll}
\hline SNR & $\mathrm{T}_{0}$ & $\mathrm{~T}_{24}$ & $\mathrm{~T}_{24} / \mathrm{T}_{0}$ & $\mathrm{~T}_{48}$ & $\mathrm{~T}_{48} / \mathrm{T}_{0}$ \\
\hline Lung & 9.27 & 6.35 & 0.68 & 2.78 & 0.30 \\
Liver & 4.32 & 3.43 & 0.79 & 9.34 & 2.16 \\
\hline
\end{tabular}

121 total cells $(76.0 \%)$, and similar percentages were obtained when the labelling was repeated $(82.1 \%$ and $80.8 \%$, range $76-82.1 \%)$. The percentages of MIRBMSCs and CS-MSCs were $83.2 \%$ and $83.8 \%$, respectively, and $72.2 \% \pm 11.3 \%$ MSCs (mean \pm standard deviation) expressed the GFP. MIRB-MSC assessment of viability showed 96 cells out of 106 total cells (90.5\%), and 109 cells out of 129 total cells (84.5\%) were viable; whereas 65 CS-MSCs were viable out of $85(76.5 \%)$ and 82 cells out of 106 total cells (77.4\%). Among MSCs, $80.9 \%$ were positive to MIRB, and $78.2 \%$ were positive to CS.

CS-MSCs were detected in the right cranial lobe of the rat (corresponding to the site of MSC injections) (Fig. 5a) sacrificed $48 \mathrm{~h}$ after MSCs injection, as shown by green fluorescence via confocal microscopy (Fig. 5b); CSMSCs were found within an area of approximately 1.3 $\mathrm{mm}^{2}$ and in $0.9 \mathrm{~mm}$ of depth. Red PFC nanoparticles were detected within the MSC cells, and they were diffusely localised in the cytoplasm, (Fig. 5c). At the histopathological examination of the liver, no MSCs were detected in the slice corresponding to the signal detected by MRI.

\section{Discussion}

The most promising clinical applications of cell therapy to pulmonary diseases are the treatment of postresection bronchopleural fistula as well as some endstage diseases like cystic fibrosis or emphysema. Topical endoscopic or transthoracic CT-guided needle injection are the best ways of delivery, overcoming some drawbacks of intravenous injection that may potentially result in cells scattering outside the target areas. Based on our previous experimental experience on large animal models, single session therapy may probably be effective, although multiple treatments can be required in more extensive diseases or airway tissue loss. Many cell therapies suffer from low engraftment, mainly due to the early immune rejection and destruction of implanted cells shortly after injection [26]. Moreover, it is difficult to effectively monitor cell delivery, distribution, engraftment and possible migration or scattering from the target site to other organs and tissues [27]. Lung parenchyma and airways represent an unexplored field of cellular therapies, although some interesting premises have been reported, both in the field of regenerative medicine and drug loading and delivery [28-30]. For effective cell 


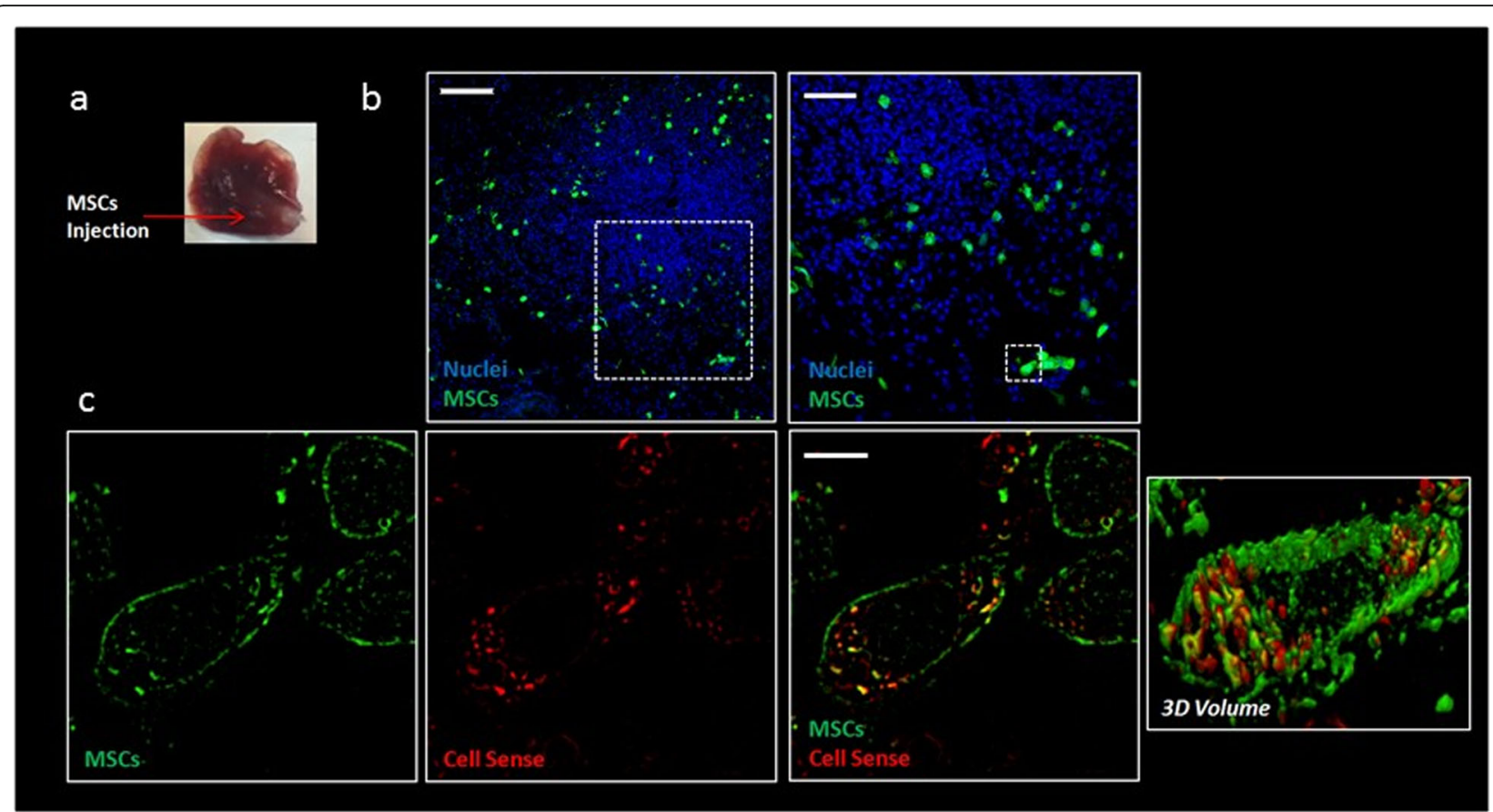

Fig. 5 Detection of green fluorescence protein (GFP) mesenchymal stromal cells (MSCs) labelled with Cell Sense Red in the cranial lobe of rat lung at $\mathrm{T}_{48}$. a Image of right cranial lobe of rat lung and indicative direction of MSC injection. $\mathbf{b}$ Confocal fluorescence image of right cranial lobe injected with Cell Sense-labelled GFP-MSCs (20x objective, scale bar $100 \mu \mathrm{m})$ and zoom on a restricted area (40x objective, scale bar $50 \mu \mathrm{m})$. The lung was counterstained with 4',6-diamidino-2-phenylindole (nuclei). c Representative detailed zoom on GFP (green) and Cell Sense-labelled (red) MSCs through structured illumination microscopy (single-plan image) and three-dimensional volume of $4 \mu \mathrm{m} z$-stack of single Cell Sense-MSC (scale bar $5 \mu \mathrm{m}$ )

therapies, it is of paramount importance to have precise data about the in vivo fate of stem cells after transplantation as biodistribution and their functions in the local microenvironment [31]. Although direct tissue biopsy of the target organ after stem cell transplantation could offer histological data, it is almost impossible to confirm stem cell presence in the biopsied tissue because they may spread over a wide range of tissues [32]. It is therefore extremely important to develop effective and reliable non-invasive techniques to track in vivo fate of transplanted cells without tissue biopsy [33]. At the moment, these techniques can be generally divided into two different categories: reporter genes and direct labelling of stem cells with several contrast agents. When stem cells are transfected with reporter genes, they can metabolise several substrates providing longterm imaging signals because reporter genes are transmitted to daughter cells; on the other hand, this method requires genetic manipulation of stem cells by viral or non-viral vectors that pose incremental risks for mutagenesis [34, 35].

Direct labelling of stem cells by fluorescent dyes, magnetic particles and gold nanoparticles have been extensively reported: when these nanoparticles are able to enter the cells and remain trapped intracellularly, excellent labelling and tracking are possible [36]; on the other hand, this method is not effective in the case of cells with low endocytic capacity and, if nanoparticles have cationic charge, this may result in unintended changes to cell viability or functionality [37].

Since the purpose of this study was to perform MSC tracking, after injection into the lungs in a rat model, a preliminary thought to the tissue background was due. Indeed many published papers have demonstrated that USPIO signal is well visible as a black hole on T2- or T2\%-weighted images. However, we also know that the lungs, as air-containing organs, show hypointensity on T2-weighted images. For this reason, we have decided to label the MSCs with two different categories of contrast media: USPIO would have given a black hole in a black background, and it would have been unhelpful to see labelled cells in the lungs; therefore, MRI acquisition for the group of rats injected with MIRB-MSCs was limited to the abdomen. On the other hand, the PFC-labelled cells would have been detectable within the lungs after the injection since they are highly concentrated after injection and with no background artefacts. Therefore, MRI acquisition for the group of rats injected with CSMSCs included the whole body. In this study, we found that stem cell labelling by USPIO and PFC did not affect 
cell vitality; in fact, we did not observe a significant decrease in cell vitality of MIRB-MSCs and CS-MSCs compared with the time of labelling or in the percentage of labelled cells. Moreover, the presence of PFC tracer was confirmed in MSC cytoplasm at post-mortem analysis. Although we evaluated the vitality and labelling stability in vitro we cannot exclude a different fate in vivo after the injection.

MSCs are usually characterised for their ability to differentiate in the osteogenic, chondrogenic, and adipogenic lineages, but they have attracted particular attention because of the anti-inflammatory and regenerative properties in the lungs, through the secretion of cytokines and extracellular vesicles [38]. Many studies have already explored the effect of the selected labelling agents (MIRB and CS) in MSCs of different origins, and they did not observe alterations on their features [3941]. For this reason, in this paper, we did focus on the fate of the cells when injected, rather than on the differentiation capabilities of labelled MSCs.

Interestingly, MRI showed the presence of CS-MSCs in the injected lung in 5/5 animals at $\mathrm{T}_{0}$, in $1 / 2$ animals at $\mathrm{T}_{24}$, and in $1 / 3$ animals at $\mathrm{T}_{48}$, suggesting a sort of linear decay. Looking at the ${ }^{19} \mathrm{~F}$ signal in the liver, $3 / 5$ of the injected animals presented a visible signal at T0, 1/2 at $\mathrm{T}_{24}$, and $2 / 3$ at $\mathrm{T}_{48}$, disclosing a similar, although more persistent, pathway compared with the lung.

A previous study demonstrated that MRI was able to detect in vitro the fluorine signal of labelled cells in pellets with a minimum number of $0.5 \times 10^{6}$ [12]. Therefore, the signal decay in the lungs can be referred to a reduction of the labelled cells. However, we cannot prove if this signal reduction over time is due to undetectable partial cell scattering to other sites or local cell death, although cell homogeneity would rather suggest cell spreading rather than selective local cell death; nevertheless, this remains a hypothesis, and further experiments are needed for clearer results.

We found the presence of high hepatic signal at time zero after injection of CS-MSCs; interestingly, it could be argued that MSCs locally injected into the lung may reach the liver via standard blood flow of the cardiac cycle. Nevertheless, the fact that $40 \%$ of the animals did not show any liver signal at time zero could be explained by cell migration to the liver only of those cells injected more centrally and close to pulmonary vessels, whereas cells injected more peripherally in the lung parenchyma may not be able to reach the systemic blood flow. Furthermore, this finding was detected only in the group of rats injected with the CS-MSCs, whereas no signal was detected in the liver of the rats injected with the MIRB-MSCs. This difference might be due to a casual scattering of the cells, depending on the site of the injection, as previously mentioned. In fact, after the death of a rat at $\mathrm{T}_{0}$, the injection was performed slightly more peripherally. Although we cannot exclude that the efficiency of the MIRB labelling was lower than the CS labelling, the possibility to identify in vitro MIRB-MSCs via fluorescence analysis has already been demonstrated. Therefore, the failure in detection of MIRB-MSCs in vivo might be caused by the fact that MIRB-MSCs remained within the lung, and MRI could not detect their signal, rather than a failure in labelling.

This study does have several limitations. First of all, the small number of rats $(n=10)$ may have affected the results. However, the $3 \mathrm{R}$ principle (replace, reduce, refine) for animal experimentation impose to reduce the number of laboratory animals to the greatest possible extent. Before starting the study, we did not know if the anaesthesia protocol performed without isoflurane would have been effective and if the rats would have lived after a lung injection. For this reason, as exploratory study, instead of increasing the number of animals for each MRI evaluation, we used the same animal as control for itself at several time points. Another limitation is that our hypothesis about MSCs detected in the liver at MRI and not at histological analysis cannot be demonstrated unless a computed tomography-guided transthoracic injection could be proposed to more precisely and homogeneously implant labelled cells in the lung parenchyma. However, to explain the discrepancy between MRI and microscopy results, we have to keep in mind that MRI and histological evaluation through fluorescence microscopy are based on different principles and provide complementary information. Indeed, MRI is an approach to the entire organ, but it does not give precise information at cellular level, whereas the histological analysis on slides of tissue via microscopy makes it possible to inspect the single organ in detail (single cells and morphology), but with the limitation of tissue manipulation (extraction, washing, fixation, and storage) and longer analysis time.

In conclusion, PFC-based emulsion provides clearer findings in an in vivo rat model compared with USPIO contrast agent, specifically when evaluating the lung parenchyma; the scattering to other solid organs (liver) was also better seen by PFC-labelled cells, likely because of the iron content within the liver. A linear decay of injected CS-MSCs was observed at $\mathrm{T}_{0}, \mathrm{~T}_{24}$ and $\mathrm{T}_{48}$ in the lung; in more than half of the experiments, CS-MSC scattering to the liver was observed, with a similar decay over time as observed in the lung.

\section{Abbreviations}

CS: Cell Sense; DAPI: 4',6-diamidino-2-phenylindole; GFP: Green fluorescence protein; MIRB: Molday ION Rhodamine B; MRI: Magnetic resonance imaging; MSCs: Mesenchymal stromal cells; PBS: Phosphate saline buffer;

PFC: Perfluorocarbon; RARE: Rapid acquisition with refocused echoes; ROI: Region of interest; SNR: Signal-to-noise ratio; USPIO: Ultrasmall paramagnetic iron oxides 


\section{Acknowledgements}

The authors thank Susan Jane West for editing the English text.

\section{Authors' contributions}

$\mathrm{SR}, \mathrm{FPa}, \mathrm{ER}, \mathrm{DG}, \mathrm{FPe}$ : conceptualisation, data curation, methodology, supervision, visualisation, original draft writing; DA, SB, FA: methodology, supervision; $L S, F B, M B, M G B$ : conceptualisation, resources, supervision. All authors read and approved the final manuscript.

\section{Funding information}

The present study was funded by the European Institute of Oncology Foundation, Milan, Italy.

\section{Availability of data and materials}

The datasets used and/or analysed during the current study are available from the corresponding author on reasonable request.

\section{Ethics approval and consent to participate}

The animal procedures were performed in accordance with the Italian laws on animal protection. Ministry of Health authorisation n.21/2017-PR released on January 16th 2017, according to the article $n .31$ of the D.Igs n.26/2014.

\section{Consent for publication}

Not applicable

\section{Competing interests}

The authors declare that they have no competing interests

\section{Author details}

${ }^{1}$ Imaging Institute of the Southern Switzerland (IIMSI), Ente Ospedaliero Cantonale (EOC), via Tesserete 46, 6900 Lugano, Switzerland. ${ }^{2}$ Facoltà di Scienze biomediche, Università della Svizzera italiana (USI), Via G. Buffi 13, 6904 Lugano, Switzerland. ${ }^{3}$ Clinica di Radiologia EOC, Istituto di Imaging della Svizzera Italiana (IIMSI), via Tesserete 46, 6900 Lugano, Switzerland. ${ }^{4}$ Neuroradiology Unit, Fondazione IRCCS Istituto Neurologico Carlo Besta, Milan, Italy. ${ }^{5}$ Neuroimmunology and Neuromuscular Diseases Unit, Fondazione IRCCS Istituto Neurologico Carlo Besta, Milan, Italy. ${ }^{6}$ Dipartimento di Medicina Veterinaria, Università degli Studi di Milano, Milan, Italy. ${ }^{7}$ Department of Health, Animal Science and Food Safety, Università degli Studi di Milano, Milan, Italy. ${ }^{8}$ Department of Thoracic Surgery, IRCCS European Institute of Oncology, Milan, Italy. ${ }^{9}$ Department of Oncology and Hemato-oncology, University of Milan, Milan, Italy. ${ }^{10}$ Department of Radiology, IRCCS European Institute of Oncology, Milan, Italy. ${ }^{11}$ Department of Neuroradiology, Fondazione IRCCS Istituto Neurologico Carlo Besta, Milan, Italy. ${ }^{12}$ CRC StaMeTec Università degli studi di Milano, Milan, Italy.

Received: 2 April 2020 Accepted: 4 August 2020

Published online: 08 October 2020

\section{References}

1. Mason C, Dunnill P (2008) A brief definition of regenerative medicine. Regen Med 3:1-5. https://doi.org/10.2217/17460751.3.1.1

2. Petrella F, Rizzo S, Borri A, Casiraghi M, Spaggiari L (2015) Current perspectives in mesenchymal stromal cell therapies for airway tissue defects. Stem Cells Int 2015:746392. https://doi.org/10.1155/2015/746392

3. Petrella $F$, Spaggiari $L$ (2018) Stem cells application in thoracic surgery: current perspective and future directions. Adv Exp Med Biol 1089:143-147. https://doi.org/10.1007/5584_2018_180

4. Pittenger MF, Mackay AM, Beck SC et al (1999) Multilineage potential of adult human mesenchymal stem cells. Science 284:143-147. https://doi.org/ 10.1126/science.284.5411.143

5. Petrella F, Toffalorio F, Brizzola S et al (2014) Stem cell transplantation effectively occludes bronchopleural fistula in an animal model. Ann Thorac Surg 97:480-483. https://doi.org/10.1016/j.athoracsur.2013.10.032

6. Petrella F, Spaggiari L, Acocella F et al (2015) Airway fistula closure after stem-cell infusion. N Engl J Med 372:96-97. https://doi.org/10.1056/ NEJMC1411374

7. Petrella $F$, Coccè V, Masia C et al (2017) Paclitaxel-releasing mesenchymal stromal cells inhibit in vitro proliferation of human mesothelioma cells. Biomed Pharmacother 87:755-758. https://doi.org/ 10.1016/j.biopha.2017.01.118
8. Petrella F, Rimoldi I, Rizzo S, Spaggiari L (2017) Mesenchymal stromal cells for antineoplastic drug loading and delivery. Medicines (Basel) 4:E87. https:// doi.org/10.3390/medicines4040087

9. Facchetti G, Petrella F, Spaggiari L, Rimoldi I (2017) Malignant pleural mesothelioma: state of the art and advanced cell therapy. Eur J Med Chem 142:266-270. https://doi.org/10.1016/j.ejmech.2017.07.063

10. Petrella F, Rimoldi I, Facchetti G, Spaggiari L (2018) Novel platinum agents and mesenchymal stromal cells for thoracic malignancies: state of the art and future perspectives. Expert Opin Ther Pat 28:813-821. https://doi.org/10. 1080/13543776.2018.1528234

11. Rimoldi I, Coccè V, Facchetti G et al (2018) Uptake-release by MSCs of a cationic platinum (II) complex active in vitro on human malignant cancer cell lines. Biomed Pharmacother 108:111-118. https://doi.org/10.1016/j. biopha.2018.09.040

12. Rizzo S, Petrella F, Zucca I et al (2017) In vitro labelling and detection of mesenchymal stromal cells: a comparison between magnetic resonance imaging of iron-labelled cells and magnetic resonance spectroscopy of fluorine-labelled cells. Eur Radiol Exp 1:6. https://doi.org/10.1186/s41747017-0010-9

13. Helfer BM, Balducci A, Sadeghi Z et al (2013) 19F MRI tracer preserves in vitro and in vivo properties of hematopoietic stem cells. Cell Transplant 22:87-97. https://doi.org/10.3727/096368912X653174

14. Chen R, Yu H, Jia ZY, Yao QL, Teng GJ (2011) Efficient nano iron particlelabeling and noninvasive MR imaging of mouse bone marrow-derived endothelial progenitor cells. Int J Nanomedicine 6:511-519. https:/doi.org/ $10.2147 /$ IJN.S16934

15. Wang P, Petrella F, Nicosia L, Bellomi M, Rizzo S (2016) Molecular imaging of stem cell transplantation for liver diseases: monitoring, clinical translation, and theranostics. Stem Cells Int 2016:4058656. https://doi.org/10.1155/2016/ 4058656

16. Choi D, Kim JH, Lim M et al (2008) Hepatocyte-like cells from human mesenchymal stem cells engrafted in regenerating rat liver tracked with in vivo magnetic resonance imaging. Tissue Eng Part C Methods 14:15-23. https://doi.org/10.1089/tec.2007.0329

17. Gaudet JM, Ribot EJ, Chen Y, Gilbert KM, Foster PJ (2015) Tracking the fate of stem cell implants with fluorine-19 MRI. PLoS One 10:e0118544. https:// doi.org/10.1371/journal.pone.0118544 eCollection 2015

18. Ahrens ET, Flores $R, X u H$, Morel PA (2005) In vivo imaging platform for tracking immunotherapeutic cells. Nat Biotechnol 23:983-987. https://doi. org/10.1038/nbt1121

19. Helfer BBM, Balducci A, Nelson AD et al (2010) Functional assessment of human dendritic cells labeled for in vivo (19) F magnetic resonance imaging cell tracking. Cytotherapy 12:238-250. https://doi.org/10.3109/ 14653240903446902

20. Heyn C, Bowen CV, Rutt BK, Foster PJ (2005) Detection threshold of single SPIO-labelled cells with FIESTA. Magn Reson Med 53:312-320

21. Stuckey DJ, Carr CA, Martin-Rendon E et al (2006) Iron particles for noninvasive monitoring of bone marrow stromal cell engraftment into, and isolation of viable engrafted donor cells from, the heart. Stem Cells 24 : 1968-1975

22. Bonetto F, Srinivas M, Heerschap A et al (2011) A novel (19) F agent for detection and quantification of human dendritic cells using magnetic resonance imaging. Int J Cancer 129:365-373. https://doi.org/10.1002/ijc. 25672

23. Canzi L, Castellaneta V, Navone S et al (2012) Human skeletal muscle stem cells anti-inflammatory activity ameliorates clinical outcome in amyotrophic lateral sclerosis model. Mol Med 18:401-411. https://doi.org/10.2119/ molmed.2011.00123

24. Fanti S, Farsad M, Battista G et al (2003) Somatostatin receptor scintigraphy for bronchial carcinoid follow-up. Clin Nucl Med 28:548-552. https://doi.org/ 10.1097/00003072-200307000-00003

25. Ahrens ET, Helfer BM, O'Hanlon CF, Schirda C (2014) Clinical cell therapy imaging using a perfluorocarbon tracer and fluorine-19 MRI. Magn Reson Med 72:1696-1701. https://doi.org/10.1002/mrm.25454

26. Qiao H, Surti S, Choi SR et al (2009) Death and proliferation time course of stem cells transplanted in the myocardium. Mol Imaging Biol 11:408-414. https://doi.org/10.1007/s11307-009-0222-3

27. Fu Y, Weiss CR, Kedziorek DA et al (2019) Noninvasive monitoring of allogeneic stem cell delivery with dual-modality imaging-visible microcapsules in a rabbit model of peripheral arterial disease. Stem Cells Int. https://doi.org/10.1155/2019/9732319 
28. Rizzo S, Petrella F, Politi LS, Wang P (2017) Molecular imaging of stems cells: in vivo tracking and clinical translation. Stem Cells Int. https://doi.org/10. $1155 / 2017 / 1783841$

29. Petrella $F$ (2018) Regenerative medicine in cardiothoracic surgery: do the benefits outweigh the risks? J Thorac Dis 10:S2309-\$2311. https://doi.org/10. 21037/jtd.2017.11.86

30. Petrella F, Spaggiari L (2018) Artificial lung. J Thorac Dis 10:S2329-S2332. https://doi.org/10.21037/jtd.2017.12.89

31. Kurtz A (2008) Mesenchymal stem cell delivery routes and fate. Int J Stem Cells 1:1-7. https://doi.org/10.15283/ijsc.2008.1.1.1

32. Kis B, Pamarthi V, Fan CM, Rabkin D, Baum RA (2013) Safety and utility of transjugular liver biopsy in hematopoietic stem cell transplant recipients. J Vasc Interv Radiol 24:85-89. https://doi.org/10.1016/j.jvir.2012.09.011

33. Lee S, Yoon HI, Na JH et al (2017) In vivo stem cell tracking with imageable nanoparticles that bind bioorthogonal chemical receptors on the stem cell surface. Biomaterials 139:12-29. https://doi.org/10.1016/j.biomaterials.2017. 05.050

34. Gu E, Chen WY, Gu J, Burridge P, Wu JC (2012) Molecular imaging of stem cells: tracking survival, biodistribution, tumorigenicity, and immunogenicity. Theranostics 2:335-345. https://doi.org/10.7150/thno.3666

35. Wang F, Dennis JE, Awadallah A et al (2009) Transcriptional profiling of human mesenchymal stem cells transduced with reporter genes for imgaing. Physiol Genomics 37:23-34. https://doi.org/10.1152/ physiolgenomics.00300.2007

36. Xu C, Miranda-Nieves D, Ankrum JA et al (2012) Tracking mesenchymal stem cells with iron oxide nanoparticle loaded poly (lactide-co-glycolide) microparticles. Nano Lett 12:4131-4139. https://doi.org/10.1021/nl301658q

37. Babic M, Horak D, Trchova M et al (2008) Poly (L-lysine)-modified iron oxide nanoparticles for stem cell labeling. Bioconjug Chem 19:740-750. https:// doi.org/10.1021/bc700410z

38. Behnke J, Kremer S, Shahzad T et al (2020) MSC based therapies-new perspectives for the injured lung. J Clin Med 9:682. https://doi.org/10.3390/ jcm9030682

39. Sibov TT, Pavon LF, Miyaki LA et al (2014) Umbilical cord mesenchymal stem cells labeled with multimodal iron oxide nanoparticles with fluorescent and magnetic properties: application for in vivo cell tracking. Int J Nanomedicine 9:337-350. https://doi.org/10.2147/IJN.S53299

40. Ribot EJ, Gaudet JM, Chen Y, Gilbert KM, Foster PJ (2014) In vivo MR detection of fluorine-labeled human MSC using the bSSFP sequence. Int $J$ Nanomedicine 9:1731-1739. https://doi.org/10.2147/JN.S59127

41. Bible E, Dell'Acqua F, Solanky B et al (2012) Non-invasive imaging of transplanted human neural stem cells and ECM scaffold remodeling in the stroke-damaged rat brain by (19)F- and diffusion-MRI. Biomaterials 33:28582871. https://doi.org/10.1016/j.biomaterials.2011.12.033

\section{Publisher's Note}

Springer Nature remains neutral with regard to jurisdictional claims in published maps and institutional affiliations.

\section{Submit your manuscript to a SpringerOpen ${ }^{\circ}$ journal and benefit from:}

- Convenient online submission

- Rigorous peer review

- Open access: articles freely available online

- High visibility within the field

- Retaining the copyright to your article

Submit your next manuscript at $\boldsymbol{\nabla}$ springeropen.com 